\title{
Radiology Quiz: Indirect Carotico-Cavernous Fistula
}

\author{
Salil Sood*, Anna Margaret Smith, Rufus Corkill, Tim Saunders, Thamir Yasen and Deepak Gupta \\ Department of ENT, Bradford Royal Infirmary, UK
}

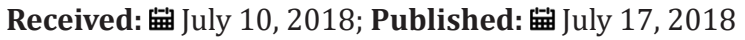

*Corresponding author: Salil Sood, ENT Fellow, Department of ENT, Bradford Royal Infirmary, UK

\section{Short Communication}

A 71-year-old lady attended ENT clinic with a nine-month history of right pulsatile tinnitus and peri-nasal pain. There was no hearing loss, vertigo or nasal complaint. Otoscopic and flexible nasendoscopy examination was entirely normal. On auscultation there was no neck bruit. An audiogram showed only a mild, highfrequency, symmetrical sensorineural hearing loss. In view of the unilateral symptoms she had an MRI (Figure 1) which was normal, and the patient was subsequently discharged. Four months later she presented as an emergency to the Ophthalmologists with sudden onset of a swollen and erythematous right eye with decreased eye movements, which was preceded by intermittent blurred vision of the affected eye. On examination her eye displayed moderate peri-orbital soft tissue swelling with chemosis and proptosis. The interorbital pressure (Normal-up to $21 \mathrm{mmHg}$ ) was between $42 \mathrm{mmHg}$ and $34 \mathrm{mmHg}$. The visual acuity of the right was $6 / 60$ or less. There was no pulsation and no bruit. A CT scan on admission showed proptosis and extraocular muscle enlargement. A provisional diagnosis of pseudotumour was made and she was treated with a lateral canthotomy to relieve the pressure, IV methyl prednisolone and IV antibiotics. As she was still symptomatic another MRI was performed (Figure 2) following which angiography was done (Figure 3).

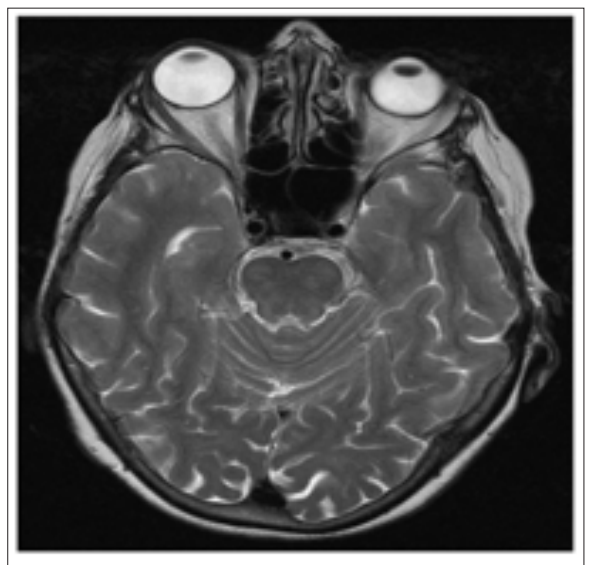

Figure 1: There is minor proptosis of the right eye with no enlargement of the superior orbital vein on an axial T2 of the brain at the level of the cavernous sinuses and orbits.

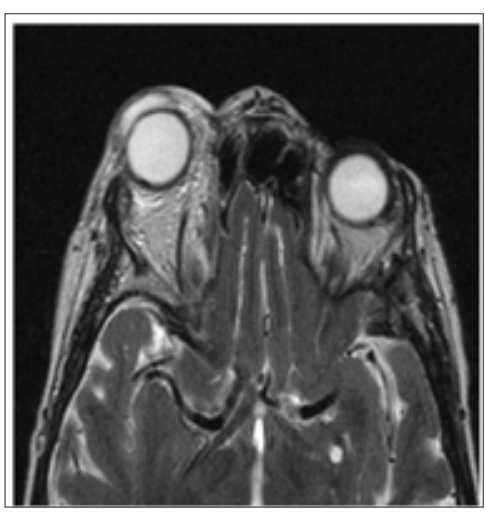

Figure 2: MRI Axial T2 images. The proptosis has markedly increased and there is oedema within retro orbital fat and congestive enlargement of the extraocular muscles. The superior ophthalmic vein has dilated due to AV shunting. The features are consistent with a carotid cavernous fistula, but conventional catheter angiography is required to demonstrate the fistula.

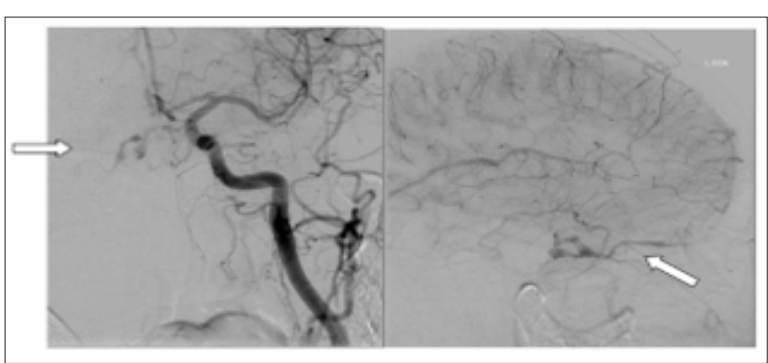

Figure 3: Pretreatment left common carotid angiogram in the AP (mid arterial phase) and lateral (late capillary phase) projections showing early venous filling through the cavernous sinus to the right superior ophthalmic vein.

\section{What is your Diagnosis?}

\section{Diagnosis: Indirect Carotico-Cavernous Fistula}

Carotico-cavernous fistulas (CCF) are abnormal communications between the carotid artery or its tributaries and the cavernous sinus. The clinical presentation was first described in the 1930's, but the anatomy was not explored until the 1970's and 1980's [1]. Dissections taught us that the cavernous sinuses are part of 
the antero-inferior group of venous sinuses. They lie on each side of the body of the sphenoid bone and extend from the superior orbital fissure to the apex of the petrous temporal bone, with an average length of $2 \mathrm{~cm}$ and width of $1 \mathrm{~cm}$. A number of structures pass through the sinus including the internal carotid artery, the abducent, trochlear and oculomotor nerves and the maxillary and ophthalmic divisions of the trigeminal nerve [2]. The sinuses have many tributaries, the most relevant of which to this discussion is the superior ophthalmic vein, as this is instrumental in the clinical presentation and radiological diagnosis of a CCF.

The pathophysiology has been explored and classified by a number of groups; the one most commonly used was devised by Barrow et al. in 1985. It was based upon the arterial supply of the fistulas which divides them into 4 categories [3]:

a) Type A fistulas are direct communications between the intra cavernous portion of the internal carotid artery and the cavernous sinus. These are otherwise known as direct CCFs and are high-flow and high-pressure fistulas.

b) Type B fistulas involve a dural shunt between branches of the internal carotid artery (ICA) and the cavernous sinus.

c) Type C fistulas involve a dural shunt between the cavernous sinus and the meningeal branches of the external carotid artery (ECA).

d) Type $D$ fistulas comprise a combination of type B and type C. Types B, C and D can all also be described as indirect CCFs and tend to have more benign disease progression.

The aetiology of CCF is in some dispute. They can be secondary to trauma, but the majority are spontaneous and of debated aetiology. The direct form is thought to occur as a result of an intra cavernous carotid artery aneurysm rupture or skull base fracture with direct tear. The indirect forms have several proposed mechanisms: One is that they are thought to form after rupture of the dural arteries that transverse the cavernous sinus. Other suggested aetiologies of the indirect CCF are that they are congenital arteriovenous malformations which start to open up, or that there is development of a lateral sinus thrombosis with recanalization [4]. Risk factors for the development of a spontaneous CCF include female gender with a 7:1 female to male ratio1, age over 50 years, collagen disorders and straining, for example during labour.

The clinical presentation of a CCF is due to the effects of arteriovenous mixing via the fistula, thus causing pressure within the cavernous sinus and its tributaries to rise and the direction of blood flow to alter. The most common presenting complaints are those of a red, swollen eye with proptosis, diplopia and ophthalmoplegia. They can also, rarely, present with pulsatile tinnitus and headache. Signs of the disease include dilated episcleral veins, chemosis, proptosis, diminished visual acuity, elevated intra-ocular pressure, and palsies of cranial nerves III and VI [5].

The treatment of CCF varies according to the individual case. Many cases of indirect fistulas undergo spontaneous resolution, so the best course of action in many cases is a watch and wait approach. However, in cases of deteriorating visual acuity, raised intra orbital pressure $(40 \mathrm{mmHg}=$ emergency, $30 \mathrm{mmHg}=$ urgent treatment) diplopia, proptosis causing corneal exposure, or when symptoms are not tolerable, intervention is necessary. In less severe CCF gamma-knife radiosurgery can be used; this causes closure of the fistula by inducing endothelial cell proliferation. High-flow fistulas require more aggressive intervention; methods used include endovascular embolization, ligation of the internal carotid artery, or open surgery to repair a tear in a traumatic CCF. Surgical intervention carries a 2-4\% risk of stroke [5].

In the case presented the patient initially presented with pulsatile tinnitus. The investigation of pulsatile tinnitus involves a thorough examination of the ear, nose and throat, audiogram, tympanometry and auscultation of the neck, retro auricular area and orbits [6]. The pathologies which should be considered are vascular neoplasms, arterio-venous malformations and other vascular anomalies. The preferred radiological investigation of pulsatile tinnitus is magnetic resonance imaging (MRI) but computed tomography (CT) scans are also acceptable. If a CT is performed it should be performed after intravenous contrast with both bone and soft tissue algorithms. Transverse sections are taken at $1 \mathrm{~mm}$ intervals through the temporal bones and skull base and 3-5mm intervals through the brain, calvaria and overlying soft tissues [7]. CT Angiography/Venography can be a valuable imaging tool in the assessment of pulsatile tinnitus [8].

The second MRI (Figure 2) scan showed marked proptosis and oedema within retro orbital fat and congestive enlargement of the extraocular muscles. The superior ophthalmic vein was dilated due to AV shunting. The features were consistent with a carotid cavernous fistula, but conventional catheter angiography was advised to demonstrate the fistula. Pre-treatment left common carotid angiogram in the AP (Figure 3) (mid arterial phase) and lateral (Figure 4) (late capillary phase) projections showing early venous filling through the cavernous sinus to the right superior ophthalmic vein. So, this case was of a type D indirect CCF which was successfully treated with endovascular embolization. Post treatment left common carotid angiogram AP (mid arterial phase) and lateral (mid arterial phase) projection showed coils in the superior ophthalmic vein and no residual shunt.

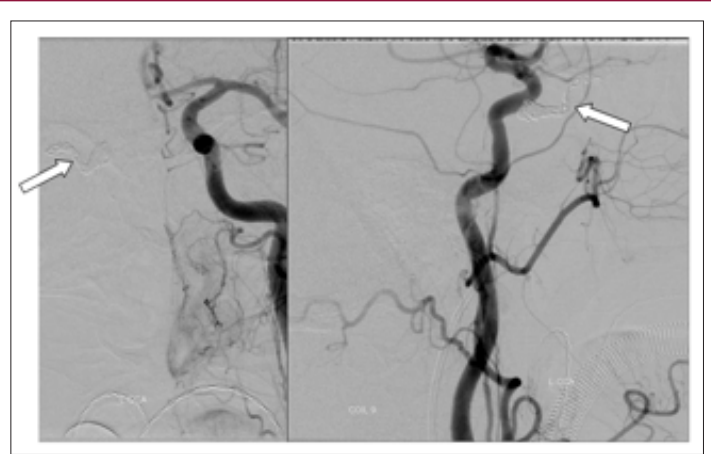

Figure 4: Post treatment left common carotid angiogram AP (mid arterial phase) and lateral (mid arterial phase) projection showing coils in the superior ophthalmic vein and no residual shunt.

The presentation involved an initial phase of intermittent pulsatile tinnitus followed by the sudden onset of an acute sight- 
threatening phase. This can perhaps be explained by the involvement of an increasing number of carotid artery branches, especially more anterior branches, leading to increasing intra cavernous pressure which eventually reached a critical level. This critical pressure no longer allowed sufficient drainage of the superior ophthalmic vein and resulted in the red, swollen eye and proptosis. At the time of the initial presentation the CCF was not sufficiently advanced to show up on the MRI. It reminds us that CCF can be an elusive diagnosis particularly in the early stages or in less severe disease. Radiological investigations like CT and MRI angiogram may miss a dural fistula either at the cavernous sinus or elsewhere, so if clinical suspicion is high and cross-sectional imaging is negative than a conventional catheter angiography is mandatory.

\section{References}

1. Nosko MG (2015) Caroticocavernous Fistula.

2. Janjua RM, Al-Mefty O, Densler DW, Shields CB (2008) Dural relationship of meckel cave and lateral wall of cavernous sinus. Nerosurg Focus 25(6): 2 .

\section{ISSN: 2574-1241}

DOI: 10.26717/BJSTR.2018.06.001421

Salil Sood. Biomed J Sci \& Tech Res

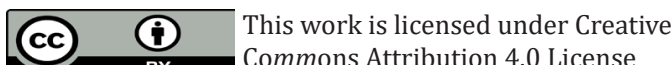

Submission Link: https://biomedres.us/submit-manuscript.php
3. Barrow DL, Spector RH, Braun IF, Landman JA, Tindall SC, et al. (1985) Classification and treatment of spontaneous carotid-cavernous sinus fistulas. J Neurosurg 62(2): 248-256.

4. Robertson A, Nicolaides AR, Taylor RH (1999) Spontaneous caroticocavernous fistula presenting as pulsatile tinnitus. J Laryngol Otol 113: 744-746.

5. Mohyuddin A (2000) Indirect carotid cavernous fistula presenting as pulsatile tinnitus. J Laryngol Otol 114(10): 788-789.

6. Lerut B, De Vuyst C, Ghekiere J, Vanopdenbosch L, Kuhweide R (2007) Post-traumatic pulsatile tinnitus: the hallmark of a direct caroticocavernous fistula. J Laryngol Otol 121(11): 1103-1107.

7. Weissman JL, Hirsh BE (2000) Imaging of tinnitus: A Review. Radiology 216(2): 342-349.

8. Krishnan A, Mattox DE, Fountain AJ, Hudgins PA (2006) CT Angiography and venography in pulsatile tinnitus: Preliminary results. Amer Journal Neuroradiology 27(8): 1635-1638.

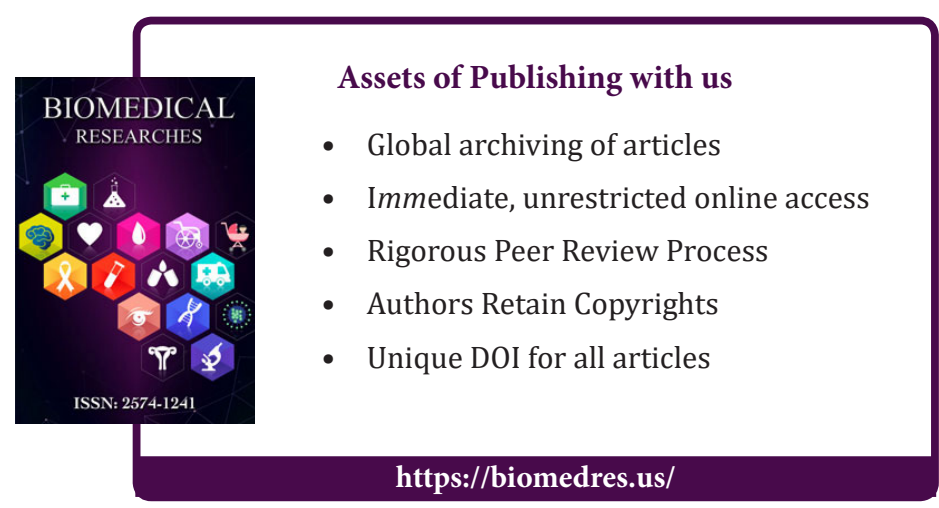

Cite this article: Salil S, Anna Margaret S, Rufus C, Tim S, Thamir Y, Deepak G. Radiology Quiz: Indirect Carotico-Cavernous Fistula. Biomed J 